\title{
USING KINETIC MONTE CARLO SIMULATIONS TO STUDY PHASE SEPARATION IN ALLOYS
}

\author{
RICHARD WEINKAMER ${ }^{\mathrm{a}, *}$, PETER FRATZL ${ }^{\mathrm{a}}$, HIMADRI S. GUPTA $^{\mathrm{a}}$, \\ OLIVER PENROSE ${ }^{\mathrm{b}}$ and JOEL L. LEBOWITZ \\ ${ }^{a}$ Max Planck Institute of Colloids and Interfaces, Department of Biomaterials, \\ 14424 Potsdam, Germany; \\ ${ }^{\mathrm{b}}$ Department of Mathematics, Heriot-Watt University, Riccarton, Edinburgh EH14 4AS, \\ Scotland, UK; \\ ${ }^{\mathrm{c}}$ Departments of Mathematics and Physics, Rutgers University, Hill Center, \\ Busch Campus, New Brunswick, 08903 New Jersey, USA
}

(Received 17 December 2003)

\begin{abstract}
We review recent extensions of the kinetic Ising model used to investigate phase separation in binary alloys. Firstly, vacancies are included to model the diffusion of the atoms on the microscopic scale more realistically. These can change the coarsening rate and the coarsening mechanism. Secondly, the lattice is allowed to deform owing to the different sizes of the atoms and the resulting misfit between precipitates and matrix. The deformability of the lattice induces long-range elastic interactions between the atoms. These change the shape, orientation, and arrangement of the precipitates. The growth of the precipitates need not follow the $R(t) \propto t^{1 / 3}$ law.
\end{abstract}

Keywords: Phase separation; Rafting; Monte Carlo simulation; Kinetic Ising model; Vacancy;

Elastic interactions

\section{INTRODUCTION}

Understanding the kinetics of phase separation in alloys is of great importance for the design of new metallic materials. Most metallic alloys are hardened by the introduction of defects such as precipitates. To predict the behaviour of alloys during processing or in service, it is essential to know the nucleation, growth, coarsening, and the stability of precipitates under various conditions of temperature and applied loads. To this end, three different approaches have been developed to model phase separation in alloys based on (i) a macroscopic modeling of sharp moving interfaces, (ii) a mesoscopic phase field description, and (iii) microscopic models at the atomic level (see Fratzl et al., 1999; Binder and Fratzl, 2001; Wagner et al., 2001; Chen, 2002; Bellon, 2003; Thornton et al., 2003 for recent reviews on phase separation and its modeling).

An important class of microscopic models is based on the kinetic Ising model which has been used since the early 1970s to describe phase separation in alloys

*Corresponding author. E-mail: weinkam@mpikg-golm.mpg.de 
(Bortz et al., 1974; Marro et al., 1975; Rao et al., 1976; Binder and Fratzl, 2001). The model consists of two types of atoms, $A$ and $B$, on a fixed lattice with periodic boundary conditions. The kinetics was described by stochastic exchanges of $A$ and $B$ atoms with a probability specified by a Hamiltonian describing the interactions between the atoms. These models are quite successful in describing nucleation and growth of precipitates (Sur et al., 1977). They also led to the discovery of dynamical scaling (Marro et al., 1979; Lebowitz et al., 1982; Fratzl et al., 1991) predicted on theoretical grounds (Binder and Stauffer, 1974).

In recent years the kinetic Ising model has been extended in different directions. Two such extensions, which give additional flexibility into modeling important details of the phase separation process, are reviewed here. The first concerns the introduction of a vacancy into the alloy, yielding in fact a ternary $\mathrm{A}-\mathrm{B}-\mathrm{V}$ system. In such models the kinetics is described more realistically by atoms jumping into neighboring vacancies. This leads to a movement of vacancies across the system which in turn has a profound influence on the phase separation process. The coarsening kinetics is strongly influenced by the interaction of the vacancy with the two phases as well as with the interface between them (Section 2.2.1). Additionally, even the mechanism of coarsening itself may change - depending on the vacancy interactions - from a situation where evaporation-condensation dominates to one where the precipitates coagulate (Section 2.2.2). In another extension of the kinetic Ising model the $A$ and $B$ atoms are permitted to have different radii, and the rigid lattice was replaced by a deformable one. This model allows to take into account elastic misfit stresses between $A$-rich and $B$-rich domains which differ in lattice spacing as a consequence of the unlike atomic radii of $A$ and $B$ atoms. Depending on the crystallographic structure and the elastic anisotropy of the lattice, anisotropic domains develop and coarsen in a way which depends on the elastic misfit, the temperature, and the alloy composition (Section 3.2.1). The effect of externally applied stresses can also be included into the model to investigate their effect on anisotropic coarsening as well as precipitate rafting (Section 3.2.2). Finally, if there is competition between attractive and repulsive forces between like atoms in the alloy, ordered precipitates may be formed. The influence of elastic misfit and of external stress on the simultaneous decomposition and ordering process can be studied in the framework of the kinetic Ising model on an elastically deformable lattice (Section 3.2.3). Sections 2.2.2 and 3.2.1 contain unpublished results.

\section{PHASE SEPARATION IN THE ABV MODEL}

\subsection{The ABV Model and Monte Carlo Simulation}

\section{Lattice}

The classical atomistic computer model for a binary alloy used in kinetic Monte Carlo (MC) simulations consists of a rigid lattice, whose sites are occupied by either an $A$ or a $B$ atom. Since most of the alloys crystallize in a body-centered cubic (BCC) or face-centered cubic (FCC) structures, these are the lattices preferentially chosen. Simulations are performed in the framework of a canonical ensemble, i.e., with a fixed number $N_{A}$ of $A$ atoms and $N_{B}$ of $B$ atoms. It is essential for the first part of the work presented here that the system is allowed to contain lattice defects, namely $N_{V}$ vacancies. To avoid finite size effects, the number of lattice sites, $N=N_{A}+N_{B}+N_{V}$, and therefore the 
linear dimensions of the lattice have to be larger than a typical length characterizing the emerging microstructure, e.g., the size of the precipitates formed. A rule of thumb is that the lattice size is at least six times larger than the precipitate size (Heermann et al., 1996). Although $N>10^{6}$ is feasible on modern computers for a study of the nucleation, growth, and coarsening of precipitates, the size of precipitates that can be studied with atomistic MC simulations is therefore still restricted to about $10 \mathrm{~nm}$. To keep the influence of the finite size of the lattice small, periodic boundary conditions are usually chosen.

\section{Hamiltonian}

The chemical short-range interactions between atoms are described phenomenologically as pair interactions between nearest neighbour (NN) atoms, with $\varepsilon_{A B}$ denoting the energy of an NN-pair of an $A$ and $B$ atom. An inclusion of interactions between atoms in more distant shells is straightforward (Weinkamer et al., 1998). The Hamiltonian of this so-called ABV model (Yaldram and Binder, 1991) is then given by:

$$
H=N_{A A} \varepsilon_{A A}+N_{B B} \varepsilon_{B B}+N_{A B} \varepsilon_{A B}+N_{A V} \varepsilon_{A V}+N_{B V} \varepsilon_{B V}+N_{V V} \varepsilon_{V V}
$$

with $N_{X Y}$ the number of NN-bonds between $X$ and $Y$ atoms, $X, Y=A, B$, or $V$. Introducing a spin variable $\sigma_{i}$, which takes the values $1,-1$, or 0 , if there is an $A$ atom, $B$ atom, or vacancy at site $i$, respectively, the Hamiltonian can be rewritten as (Vives and Planes, 1993):

$$
H=H_{0}+K \sum_{\langle i j\rangle}^{N N} \sigma_{i}^{2} \sigma_{j}^{2}+J \sum_{\langle i j\rangle}^{N N} \sigma_{i} \sigma_{j}+U \sum_{\langle i j\rangle}^{N N}\left(\sigma_{i}^{2} \sigma_{j}+\sigma_{i} \sigma_{j}^{2}\right),
$$

where $H_{0}$ is constant independent of the configuration of atoms on the lattice and

$$
\begin{aligned}
K & =\frac{1}{4}\left(\varepsilon_{A A}+\varepsilon_{B B}+2 \varepsilon_{A B}\right)+\varepsilon_{V V}-\varepsilon_{A V}-\varepsilon_{B V}, \\
J & =\frac{1}{4}\left(\varepsilon_{A A}+\varepsilon_{B B}-2 \varepsilon_{A B}\right), \\
U & =\frac{1}{4}\left(\varepsilon_{A A}-\varepsilon_{B B}\right)-\frac{1}{2}\left(\varepsilon_{A V}-\varepsilon_{B V}\right) .
\end{aligned}
$$

For the case of a single vacancy, $N_{V}=1$, - the case considered in the following - the second term on the right side of Eq. (2) takes a constant value,

$$
\sum_{\langle i j\rangle}^{N N} \sigma_{i}^{2} \sigma_{j}^{2}=Z\left(\frac{N}{2}-1\right)
$$

$Z$ being the coordination number of the lattice. From the five independent pair interactions, $\varepsilon_{A A}, \varepsilon_{A B}, \varepsilon_{B B}, \varepsilon_{A V}$, and $\varepsilon_{B V}$, only two independent parameters, $J$ and $U$, 
remain. Their role within the model can be better understood, writing the Hamiltonian in the form (Weinkamer and Fratzl, 2003)

$$
H=H_{0}^{\prime}-2\left(J N_{A B}+U N_{A V}\right),
$$

with $H_{0}^{\prime}$ denoting another constant. The ordering energy $J$ describes the interaction between the atoms and, therefore, determines the phase diagram of the system. For $J<0$ the systems tend to separate into $A$-rich and $B$-rich phases, $J>0$ leads to ordering on the lattice. The presence of a single vacancy, i.e., a vacancy concentration $c_{V} \approx 10^{-6}$, does not significantly change the phase diagram (Fratzl and Penrose, 1994). The interaction of the vacancy with the atoms is specified by the asymmetry parameter $U$ (Kikuchi and Sato, 1972). $U>0$ means that the vacancy prefers $A$ atoms in its NN shell. The ordering energies $J^{(k)}$ - the superscript $(k)$ denotes the $k$ th neighbour shell for the case of interactions beyond the nearest neighbour shell - can be determined by means of diffuse scattering experiments (Schweika, 1998) and using the so-called inverse Monte Carlo method (Gerold and Kern, 1987). It was shown that the obtained $J^{(k)}$ is not unique and that many-body interactions have to be included for a good reproduction of real phase diagrams (Wolverton et al., 1997). Values for the asymmetry parameter $U$ can be estimated from the difference of the pure metal cohesive energies, the vacancy formation energies, or the vacancy migration energies (Roussel and Bellon, 2001). These estimates often lead to quite large ratios, $|U / J|>1$.

\section{Dynamics}

For the dynamical interpretation of a MC simulation, it is important to use a realistic mechanism to model the elementary changes in the atomic configuration. In early MC simulations using models, which did not include vacancies, the time evolution of the system was modeled by exchanges between atoms of different type (Kawasaki dynamics, abbreviated K-dynamics). In alloys, it is well-known that changes in the atomic configuration occur via a jump of an atom into a neighboring vacancy. The first simulations using vacancy dynamics (V-dynamics) were performed in the early 90s (Yaldram and Binder, 1991). An unrealistically high vacancy concentration of $16 \%$ chosen in these works concealed the new physics which is associated with the introduction of vacancies, and which is presented below.

\section{Exchange Probabilities}

The general principles of MC simulations can be found in Binder (1997) and Binder and Heermann (2002) and need not be repeated here. For our problems the state of the system is defined by a complete set of the spin variables, $\boldsymbol{x}_{l}=\left\{\sigma_{i}, i=1, \ldots, N\right\}$. In a kinetic MC simulation the aim is to construct a chain of states $\ldots, \rightarrow x_{l} \rightarrow x_{l+1} \rightarrow x_{l+2} \rightarrow, \ldots$, corresponding to a temporal trajectory in phase space, where on the long run the different states appear with the statistical weights given by the Boltzmann distribution. A sufficient condition for achieving this distribution is that the transition probabilities $W\left(\boldsymbol{x}_{l} \rightarrow \boldsymbol{x}_{l^{\prime}}\right)$ for passing from state $\boldsymbol{x}_{l}$ to $\boldsymbol{x}_{l^{\prime}}$ satisfy the detailed balance condition,

$$
\frac{W\left(\boldsymbol{x}_{l} \rightarrow \boldsymbol{x}_{l^{\prime}}\right)}{W\left(\boldsymbol{x}_{l^{\prime}} \rightarrow \boldsymbol{x}_{l}\right)}=\exp \left[-\Delta E / k_{B} T\right]
$$


where $k_{B}$ is the Boltzmann's constant and $\Delta E$ is the "energy difference" (see below). Since Eq. (6) does not define the probability $W\left(\boldsymbol{x}_{l} \rightarrow \boldsymbol{x}_{l^{\prime}}\right)$ uniquely, different "rules" have been proposed, like the Metropolis rule,

$$
W\left(\boldsymbol{x}_{l} \rightarrow \boldsymbol{x}_{l^{\prime}}\right)= \begin{cases}\frac{1}{\tau_{0}} \exp \left[-\Delta E / k_{B} T\right] & \text { if } \Delta E>0 \\ \frac{1}{\tau_{0}} & \text { if } \Delta E \leq 0\end{cases}
$$

or, the Glauber rule,

$$
W\left(\boldsymbol{x}_{l} \rightarrow \boldsymbol{x}_{l^{\prime}}\right)=\frac{1}{\tau_{0}} \frac{\exp \left[-\Delta E / k_{B} T\right]}{1+\exp \left[-\Delta E / k_{B} T\right]} .
$$

The factor $1 / \tau_{0}$ corresponds to an attempt frequency and defines the physical time in the simulation. Our physical understanding of how diffusion proceeds in crystalline solids allows only a very limited number of "moves" of the system, i.e., only very few $W\left(\boldsymbol{x}_{l} \rightarrow \boldsymbol{x}_{l^{\prime}}\right)$ are non-zero. More precisely, only exchanges between a vacancy and a neighbouring atom are allowed (vacancy dynamics). The choice of the correct elementary allowed moves of the system crucial for the results obtained and can therefore be seen as the Achilles heel of a kinetic MC simulation.

In Eqs. (6)-(8), $\Delta E$ denotes the jump-energy of the exchange vacancy/atom. Usually $\Delta E$ is calculated as the difference between the energy of the final state and the initial state, $\Delta E=H_{\text {final }}-H_{\text {initial }}$. An alternative is to introduce an energy barrier, which separates these two states and which the atom has to overcome in changing sites. The jump-energy $\Delta E$ is then taken to be the barrier energy minus the energy of the initial state (Abinandanan et al., 1998; Bellon, 2003). The latter scenario is more realistic, and first steps to use a parameter set specific for a given alloy system obtained by the embedded-atom-method have been undertaken (Zhao et al., 1996; Le Bouar and Soisson, 2002). Normally a much simpler model is used, where a constant energy barrier is considered, which is greater than all possible states of the system, and $\Delta E$ is calculated in a broken bond approximation (Abinandanan et al., 1998). Another proposal was to introduce a sensitivity to the local environment in a simple way. The energy of the barrier, $E_{B}$, was proposed to be (Rautiainen and Sutton, 1999),

$$
E_{B}=\frac{H_{\text {initial }}+H_{\text {final }}}{2}+C
$$

where a fixed value for $C$ is chosen such that all activation energies are positive. For the relative probability $\alpha_{i}$ that the vacancy jumps to its neighbour $i$, one then obtains

$$
\alpha_{i}=\frac{\exp \left[-\left(H_{\text {final }}^{i}-H_{\text {initial }}^{i}\right) / 2 k_{B} T\right]}{\sum_{j \in N N} \exp \left[-\left(H_{\text {final }}^{j}-H_{\text {initial }}^{j}\right) / 2 k_{B} T\right]},
$$

which is very close to the result one gets using the Metropolis rule and neglecting an energy barrier, but with a temperature multiplied by a factor 2. Summarizing the 
influence of the jump-energy evaluation is often nontrivial, as was demonstrated for the case of diffusion in an ordered alloy (Weinkamer et al., 1998), and deserves attention. Since a direct comparison between MC simulations and experimental results have high priority (Pareige et al., 1999; Weinkamer et al., 1999), all attempts to make the modeling more realistic are of great importance.

\section{Time Increments}

Following the standard Metropolis algorithm, an exchange between the vacancy and a randomly chosen $\mathrm{NN}$-atom is carried out with the probability given by Eq. (7). The time unit is then usually defined as one Monte Carlo step (MCS), i.e., one attempted exchange per lattice site. The Metropolis algorithm has the disadvantage that the rejection rate drastically increases at low temperatures, e.g., when the vacancy is trapped in a low-energy configuration. This waste of computing time can be avoided by employing the residence time algorithm of Bortz et al. (1975), where it is ensured that the vacancy performs a jump at each attempt. The incrementation of the total time corresponding to an exchange depends then on all the probabilities of possible vacancy jumps. A "trapped" vacancy would be freed again at the expense of a large time increment. The mean residence time and the simulation results are unaffected by the way the time is incremented (Novotny, 1995; Weinkamer et al., 1998). The idea of residence algorithms was extended to higher-order algorithms, which also considers in the time incrementation also the possibility that the vacancy jumps straight back to its previous position (Athenes et al., 1997; Rautiainen et al., 1999). The conversion of computer time measured in MCS to physical time measured in seconds is accomplished by giving $\tau_{0}$ the value necessary to make the diffusivity of the model equal to the experimentally measured value of the diffusivity (Abinandanan et al., 1998; Weinkamer et al., 1999).

\subsection{Influence of the Vacancy Dynamics on Phase Separation}

The influence of the vacancy mechanism on the simulation results of phase separation in the kinetic Ising model is demonstrated giving two examples: first, we compare results obtained with Kawasaki and vacancy dynamics, where the single vacancy in the system does not energetically distinguish between the two different types of atoms, $A$ and $B$. This corresponds to the case $U=0$ for the asymmetry parameter in Eq. (2), which further simplifies the Hamiltonian of Eqs. (2) and (5) to the standard Ising form,

$$
H=H_{0}+J \sum_{\langle i j\rangle}^{N N} \sigma_{i} \sigma_{j}=H_{0}^{\prime}-2 J N_{A B} .
$$

Secondly, the influence of $U$ is studied choosing values $U \neq 0$. The two different results can be best demonstrated with different microstructure morphologies: an interconnected and intertwined structure at a solute concentration $c=0.5$ and isolated precipitates at $c=0.1$, respectively. 


\subsubsection{The Vacancy Dynamics Influences the Coarsening Rate of the Precipitates}

Starting with the 1970s (Bortz et al., 1974) phase separation in a binary alloy was studied using the kinetic Ising model with Kawasaki dynamics - abbreviated in the following as $K$-dynamics and using a subscript $K$. Modeling the elementary diffusion step more realistically as an exchange between vacancy and atom ( $V$-dynamics), brought as a premature conclusion that the actual diffusion mechanism is only of minor importance for the process of phase separation. Confirmed by numerous simulations, this is true as far as the developing domain structure is concerned. Snapshot pictures, or the spherically averaged pair correlation function and its Fourier transform, the structure function $S(\boldsymbol{k}, t)$, which is experimentally accessible with scattering methods, do not allow one to discriminate whether $K$ - or $V$-dynamics were used in the simulations. A small difference can be observed during circa the first $100 \mathrm{MCS}$ due to the fact that the vacancy acts locally, it takes some time till the vacancy visited all parts of the system (Soffa et al., 2003). For later stages the kinetic Ising model showed dynamical scaling (Marro et al., 1979; Fratzl et al., 1991) with identical scaling functions for both dynamics. The spherically averaged pair correlation function $g(r)$ can be written as,

$$
g(r, t)=g_{0}(T) h(r / R(t)) .
$$

where $R$, the first zero of $g(r)$, is a measure of the domain size of the two-phase structure; $T$ denotes the temperature. The scaling function $h$ was not only independent of the dynamics used, but also the same for different lattices ( $\mathrm{sc}=$ simple cubic, BCC, FCC) and almost unchanged for different temperatures (Fratzl et al., 2000).

Although the morphologies were all but identical, the coarsening rates were quite different for simulations using either $K$ - or $V$-dynamics. In all the plots in Fig. 1 , the domain size $R$ is shown as a function of $t^{1 / 3}$. Especially at low temperatures, the rate of coarsening was significantly enhanced in the case of $V$-dynamics. For temperatures close to $T_{c}$, however, this difference vanished. The straight lines correspond to fits using the formula proposed by Huse (Huse, 1986),

$$
R(t)=R_{0}+(\lambda t)^{1 / 3}
$$

The quality of the fit was excellent for all different temperatures and lattices. The difference between the two dynamics is most noticeable when comparing the temperature dependence of the prefactor $\lambda$. For $K$-dynamics $\lambda_{K}$ decreased with decreasing temperature. Since coarsening proceeds via a diffusion of monomers for $K$-dynamics, this behaviour can be well understood as due to the drastic reduction of monomers at low temperatures. For $V$-dynamics, however, $\lambda_{V}$ increased with decreasing temperature. The ratio $\lambda_{V} / \lambda_{K}$ for all the data (5 different temperatures, 4 different lattices) satisfied the relation,

$$
\lambda_{V} / \lambda_{K}=\mu \exp \left(\nu Z J / k_{B} T\right)
$$

with $Z$ the coordination number of the lattice, and $\mu=0.082$, and $v=1.81$ obtained by fitting the data. The right-hand side of Eq. (14) with $v=2$ corresponds to the low-temperature approximation for the equilibrium concentration of monomers in the matrix. 


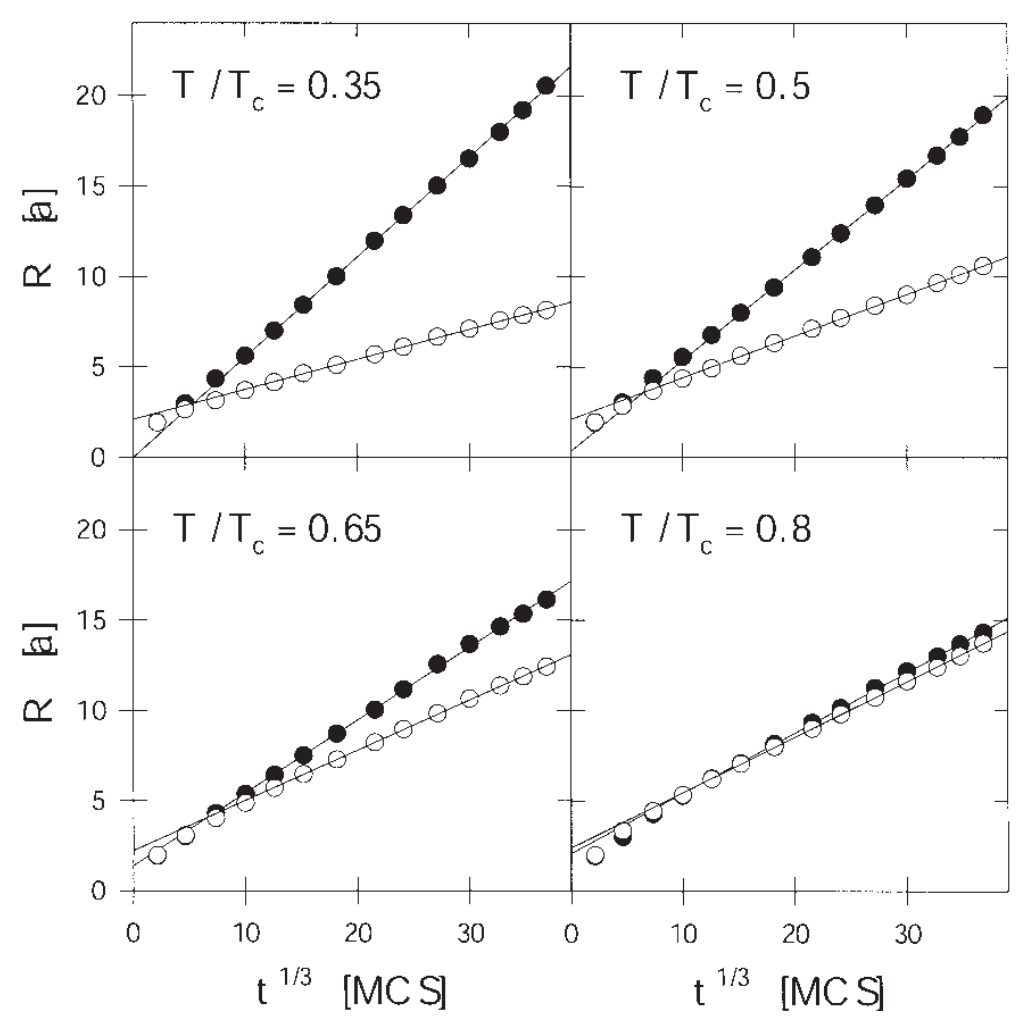

FIGURE 1 First zero $R$ of the spherically averaged pair correlation function $g(r)$ for a BCC lattice and four different temperatures, plotted as a function of $t^{1 / 3}$, where $t$ is time in MCS. $R$ is given in units of the cubic lattice constant $a, c=0.5$. Black disks represent vacancy and circles Kawasaki dynamics. The straight lines are fits with Eq. (13) for all data points $t \geq 2000$ MCS.

A theoretical understanding of these results was attempted in Fratzl et al. (2000). An important point in the argumentation is that the vacancy is located preferentially at domain interfaces, because there it breaks an energetically unfavourable bond between an $A$ and a $B$ atom (Fig. 2). At the interface, the vacancy supports interface diffusion and leads to the enhanced rate of phase separation. At higher temperatures, the binding to the interface gets smaller (Fig. 2), and the vacancy "loses time" roaming in one of the two phases. This explains qualitatively the decrease of $\lambda_{V}$ with increasing temperature. The importance of interface diffusion is reasonable in the case when the two-phase microstructure is interconnected and most of the interface can be reached via paths on the interface itself. This is surely the case for an equal concentration of $A$ and $B$ atoms, $c=0.5$. Surprisingly, the effect of an enhanced coarsening rate in the case of $V$-dynamics turned up also in the case of isolated precipitates, although not so pronounced.

\subsubsection{Coarsening by Coagulation of Precipitates or via Evaporation and Condensation of Atoms?}

The importance of the asymmetry parameter $U$ (Eqs. (2) and (3)) on the kinetic path of the model alloy evolution was realized only recently: for the case of diffusion 


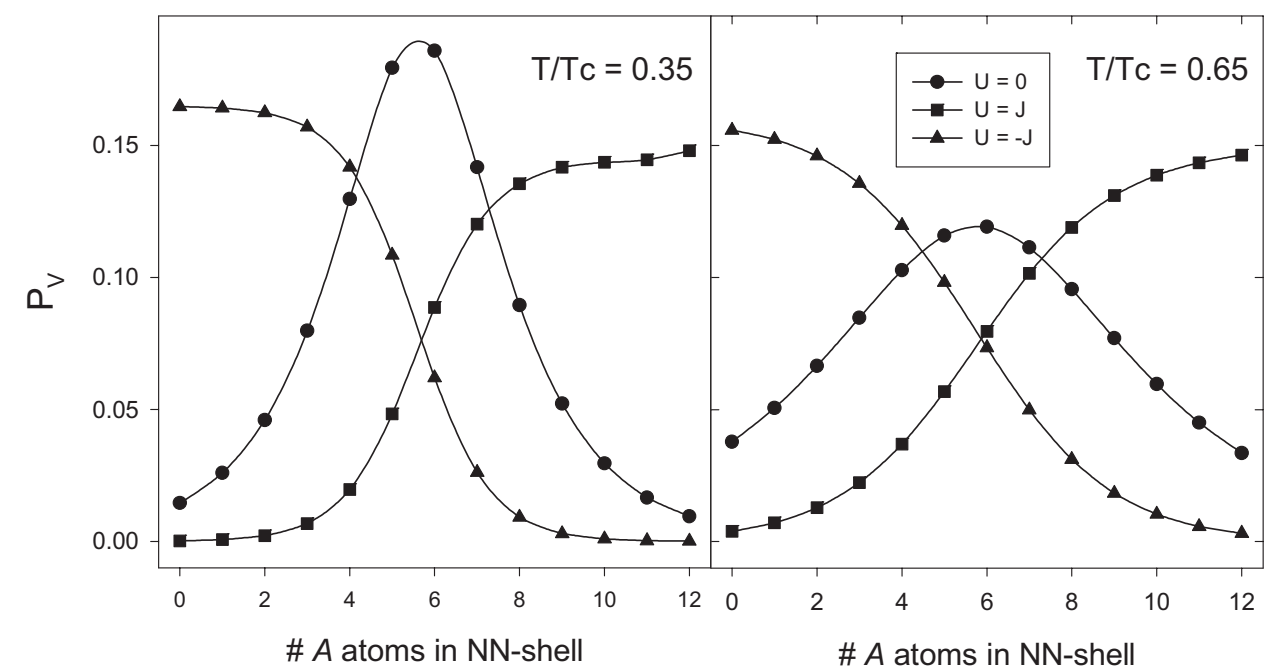

FIGURE 2 Probability $P_{V}$ of finding a specific number of solute $A$ atoms in the first nearest-neighbour shell of the vacancy during phase separation. The probability is normalized by the number of sites available with a given occupation of the NN-shell. Sites with a low (high) number of $A$ atoms correspond to a site in the matrix (precipitate), the interface sites are characterized by an approximately equal number of $A$ and $B$ atoms $(\approx 6)$. The vacancy prefers a different environment depending on the asymmetry parameter $U$ (Eqs. (2) and (3)).

(Weinkamer et al., 1999), nucleation (Soisson and Martin, 2000), phase separation (Athenes et al., 2000; Roussel and Bellon, 2001; Weinkamer and Fratzl, 2003), and ordering (Athenes et al., 1996; Porta et al., 1999). The value of $U$ affects the preferred location of the vacancy, as demonstrated in Fig. 2. For $U=0$ the vacancy prefers a nearest-neighbour shell occupied roughly by the same number of $A$ and $B$ atoms, i.e., the interface between matrix and precipitates. In simulations with $U=J$, the vacancy is preferentially inside the precipitates, while for $U=-J$ the vacancy stays most of the time inside the matrix. For higher temperatures, the vacancy path becomes less restricted to either the precipitates, the matrix, or the interface.

Fratzl and Penrose were the first who recognized the effect of the vacancy behaviour on the coarsening mechanism (Fratzl and Penrose, 1997). In the framework of the Lifshitz-Slyozov-Wagner (LSW) theory, it is assumed that coarsening occurs via a migration of single precipitate atoms, which evaporate from smaller precipitates and finally condense on larger ones - therefore it is often referred to as evaporationcondensation mechanism. The result is a growth of larger precipitates at the expense of smaller ones. An alternative mechanism is that precipitates move as a whole. When they meet, they coalesce, again with the result of a coarsening microstructure. The fact that this latter coagulation mechanism can act during coarsening, was demonstrated in a simulation with the following artificial setting (and $U=0$ ): a single, small precipitate of $A$ atoms was put in a box with only $B$ atoms, the boundary sites of the box filled with fixed $A$ atoms. The small precipitate moved around and eventually disappeared by coagulation with the boundary before it was evaporated (Fratzl and Penrose, 1997).

To get a quantitative measure of the efficiency of the two different mechanisms during coarsening, we started from a microstructure, prepared by a preliminary simula- 

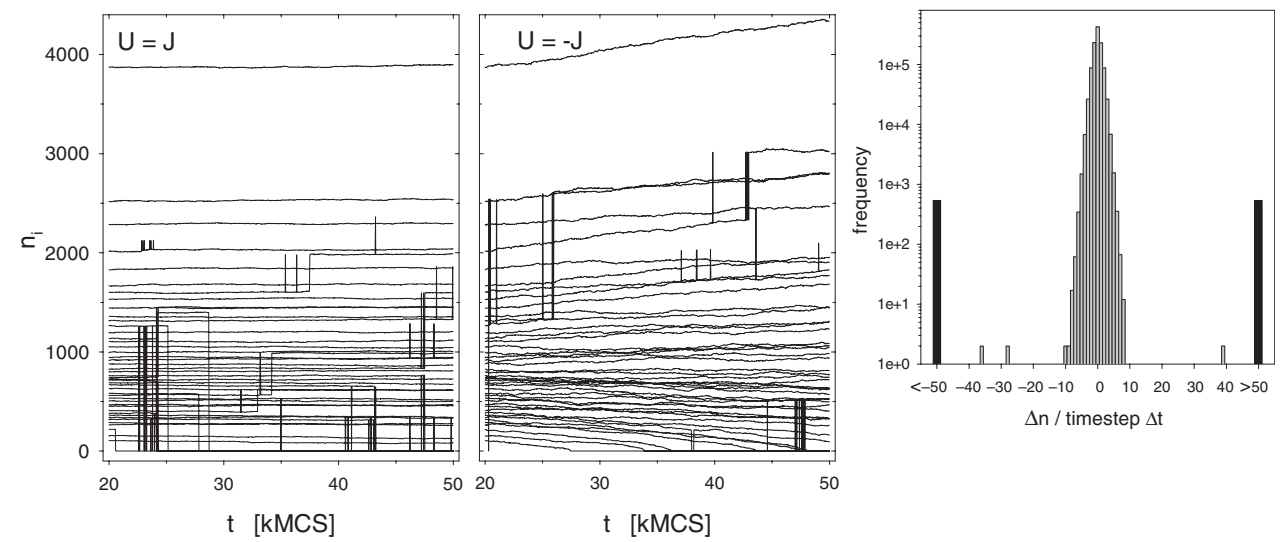

FIGURE 3 Size of individual precipitates as a function of time using vacancy dynamics with $U=J$ (left) and $U=-J$ (middle) at $T / T_{c}=0.35$ starting from the same initial configuration. For clarity trajectories of only every fifth precipitate when ordered by size are drawn. The analysis of the size changes $\Delta n$ of individual precipitates (right) during two measurements separated by $\Delta t=20 \mathrm{MCS}$ shows either small changes $(|\Delta n|<12)$ or very large changes $(|\Delta n|>50)$ corresponding to the action of different coarsening mechanisms.

tion, comprising more than 200 precipitates, the largest containing close to 4000 atoms. Monitoring the size evolution of all the precipitates in the system (Roussel and Bellon, 2001), we obtained plots like those shown in Fig. 3 for all the four different dynamics under investigation, $K$-dynamics and $V$-dynamics with $U=0, \pm J$ (Weinkamer and Fratzl, 2003). During the timestep between two measurements, the size of each precipitate changed either only by a small amount of less than about 12 atoms, or rather dramatically by more than 50 atoms (Fig. 3, right). Small changes in the size of a precipitate are due to the evaporation and condensation of monomers and small clusters (LSW mechanism). Whereas the events involving large changes and which are displayed as jumps in the trajectories in Fig. 3, has to be interpreted as coalescence events. The total increase of the mean precipitate size $\langle\Delta n\rangle$ could therefore be separated into contributions from the LSW and coagulation mechanisms, $\langle\Delta n\rangle=\langle\Delta n\rangle_{\mathrm{LSW}}+\langle\Delta n\rangle_{\mathrm{COAG}}$.

Figure 4 shows the result for the case $U=J$, with a rather smooth contribution for the LSW mechanism, but a jagged contribution - especially at higher temperatures - for the coagulation mechanism. To quantify the predominance of a coarsening mechanism, we computed the ratio $\varepsilon \equiv\langle\Delta n\rangle_{\mathrm{COAG}} /\langle\Delta n\rangle$ or a mean value $\bar{\varepsilon}$ respectively, since $\varepsilon$ slowly decreases with larger precipitate sizes. At low temperature $\left(T / T_{c}=0.35\right)$ and $U=J$, we obtain $\bar{\varepsilon}_{U=J}=0.92$ for times between 50 and $150 \mathrm{kMCS}$. In contrast for $U=-J, \bar{\varepsilon}_{U=-J}$ takes only a value of 0.15 (Weinkamer and Fratzl, 2003).

This change of the predominant coarsening mechanism with different values for $U$ can be understood as the result of a completely different vacancy path. In the case of $U=J$ the vacancy stays most of the time inside the precipitate, just leaving and reentering the precipitate at varying locations, which leads to a overall shift of the whole precipitate. The higher mobility of the precipitate enhances the probability of a coalescence event, resulting in a predominant coagulation mechanism. In contrast, for $U=-J$ the vacancy path is mainly confined to sites in the matrix, where it enhances the mobility of the monomers and small clusters of precipitate atoms, which are dissolved in the matrix. As a result coarsening occurs by the LSW mechanism. The 


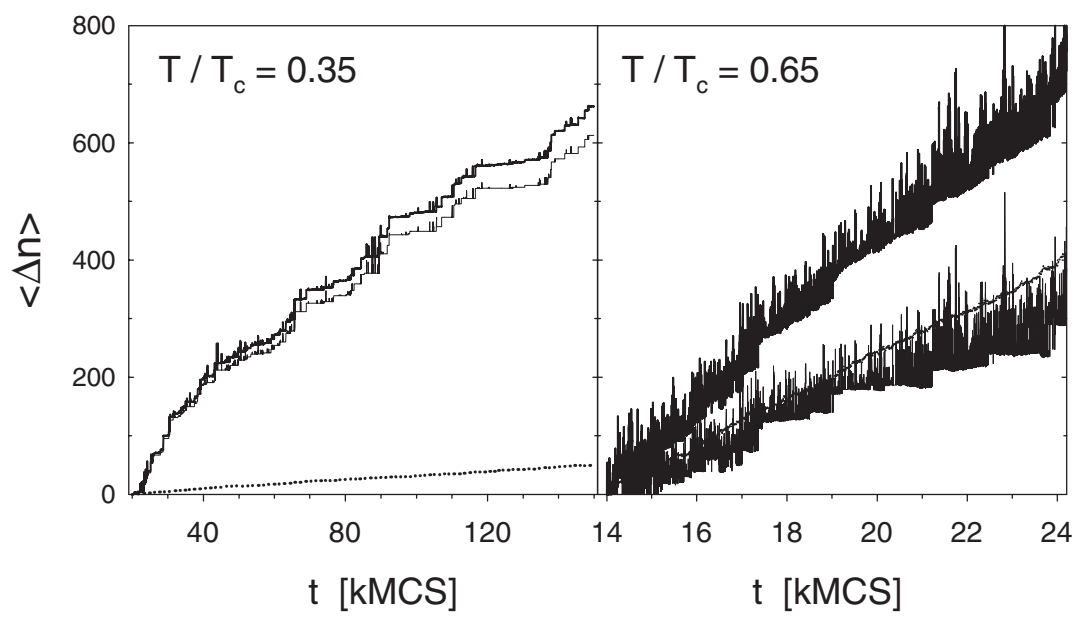

FIGURE 4 Time evolution of $\langle\Delta n\rangle$, the increase of the mean precipitate size $\langle n\rangle$ with respect to its initial value, for $U=J$ and two different temperatures (thick line). The dotted (smoother) line shows the contribution of the LSW mechanism to the overall increase, the thin (jagged) line the contribution of the coagulation mechanism, $\langle\Delta n\rangle=\langle\Delta n\rangle_{\mathrm{LSW}}+\langle\Delta n\rangle_{\mathrm{COAG}}$. The time interval of observation was chosen to have a similar increase in $\langle\Delta n\rangle$ for the two temperatures.

special case of a vacancy attracted to the interface (Mukherjee and Cooper, 1998), $U=0$, showed significant distributions for both coarsening mechanisms, $\bar{\varepsilon}_{U=0}=0.63$, leading to the highest coarsening rate of all cases under investigation.

At a higher temperature, $T / T_{c}=0.65$, the importance of the coagulation mechanism is significantly reduced. The corresponding values for $\bar{\varepsilon}$ (averaged in this case between 14 and $41 \mathrm{kMCS}$ ) are: $\bar{\varepsilon}_{U=J}=0.42$ (Fig. 4), $\bar{\varepsilon}_{U=-J}=0.16$, and $\bar{\varepsilon}_{U=0}=0.28$. These lower values for $\bar{\varepsilon}$ are in agreement with theoretical considerations (Fratzl and Penrose, 1997), and can be understood as the result of a higher solubility of precipitate atoms in the matrix and a less restricted vacancy path (Fig. 2).

The finding of two different coarsening mechanisms active during phase separation in a model alloy naturally gives rise to the question, whether the predominant coarsening mechanism can be determined experimentally. In real alloys typical values for the asymmetry parameter $U$ are comparable with the value chosen in the simulations (Roussel and Bellon, 2001). Using in situ electron microscopy the movement and coalescence of precipitates are in principle observable, but the samples have to be quasitwo-dimensional. Since the emerging microstructure characterized by the scattering function $S(\boldsymbol{k}, t)$ is undistinguishable in the simulations regardless of the predominant coarsening mechanism, measurements of $S(\boldsymbol{k}, t)$ using small-angle X-ray scattering (SAXS) do not help in discriminating coarsening mechanisms. A more promising approach seems to consider the coarsening exponent $\alpha, R(t) \propto t^{\alpha}$, from a measurement of the growth of the mean precipitate size. In contrast to the classical LSW growth law $R(t) \propto t^{1 / 3}$, a predominant coagulation mechanism should result in a coarsening exponent of $1 / 5$ instead of $1 / 3$ (Binder and Stauffer, 1974; Fratzl and Penrose, 1997). With $\mathrm{MC}$ simulations, which are admittedly restricted to a short time range and are therefore not well suited to measure "asymptotical" growth exponents, the predicted change in the exponent could not be verified satisfactorily. All the simulation data could be well fitted using Eq. (13). Hence we proposed the use of coherent X-rays from high-brilliance synchrotron sources and to perform a X-ray photon correlation spectroscopy 
(XPCS) experiment (Lengeler, 2001) to discriminate between coarsening mechanisms (Weinkamer and Fratzl, 2003). Measuring the time-correlations of intensity fluctuations - so-called speckles - the XPCS technique allows investigations of dynamic processes on a nanometer scale. Performing a computer XPCS experiment and analyzing the intensity fluctuations using fluctuation analysis (FA) (Kantelhardt et al., 2001) showed that a measurable quantity - the fluctuation exponent - as a function of length of the scattering vector, $|\boldsymbol{k}|$ was different depending on the predominant coarsening mechanism (Weinkamer and Fratzl, 2003). In a recent XPCS experiment, the same differences were found in experiments on the alloys Al-6 at. $\% \mathrm{Ag}$ and Al-9 at. $\% \mathrm{Zn}$ under conditions comparable to the simulation. These measurements indicate that coarsening in Al-6 at.\% Ag proceeds predominately via the classical LSW mechanism, while in Al-9 at. \% Zn the coagulation mechanism is prevailing (Stadler et al., 2003).

A last interesting effect due to the asymmetry parameter $U$ should be mentioned. For the modified solubility of solute $A$ atoms in the $B$-rich matrix (or of $B$ atoms in the $A$ precipitates) due to the curved interface, Roussel and Bellon found good agreement with the Gibbs-Thomson law in the case $U=0$ after short-simulation times. For $U \neq 0$, however, the solubility deviated significantly from the theoretical value (Roussel and Bellon, 2001).

\subsubsection{The Role of the Vacancy in Other Processes}

The new physics which arises from the introduction of the vacancy can be observed also in other processes. We want to mention here only some examples. The case of ordering and ordered precipitation, i.e., essentially $J>0$ in Eq. (2), has been studied extensively by a group from Spain (Vives and Planes, 1992; Porta and Castan, 1996; Frontera et al., 1997; Porta et al., 1999). Having more than one vacancy in the system, they also investigated the influence of vacancy interactions ( $K \neq 0$ in Eq. (2)) (Porta et al., 1997). The dissolution of precipitates when heated above the solubility line occurred depending on the temperature either by an evaporation of atoms or by a process resembling an explosion (Zizak et al., 1997). Starting from a completely phase separated initial configuration, i.e., pure $A$ and $B$ domains separated by a planar interface, the disordering at $T=\infty$ occurred via a different path when using vacancy dynamics compared to Kawasaki dynamics (Aspelmeier et al., 2001). When the attainment of equilibrium was prevented by an external field, the precipitates grew logarithmic in time. A new defined order parameter satisfied dynamical scaling (Schmittmann and Thies, 2002). Using the master equation approach and a mean field assumption, Puri formulated a phase field model to study vacancy-mediated phase separation (Puri, 1997). He found a growth law $R(t) \propto t^{1 / 3}$ for the size of the precipitates. Dynamical scaling is fulfilled (Puri and Sharma, 1998; Iwai, 1999) and differences to results obtained by Kawasaki dynamics were found (Puri and Sharma, 1998).

\section{PHASE SEPARATION ON AN ELASTICALLY DEFORMABLE LATTICE}

\subsection{The Extended Ising Model}

Up to now only chemical interactions between the atoms were considered. The system is then completely determined by the set $\left\{\sigma_{i}, i=1, \ldots, N\right\}$, which defines, how the rigid 
lattice is occupied by the different types of atoms. For the investigation of the influence of a lattice misfit between the $A$-rich and $B$-rich phases on phase separation, we used an extended atomistic model, where also long-range elastic interactions are included (Fratzl and Penrose, 1995). Displacements $v(\boldsymbol{r})$ of the atoms from sites of the undistorted lattice $\boldsymbol{r}$ are allowed. They are assumed to be small enough that a one-to-one mapping between atoms and these regular lattice sites still holds, i.e. the lattice remains coherent. Furthermore, the small displacements justify the use of the harmonic approximation for the interaction between the atoms. In the three-dimensional model each pair of $\mathrm{NN}$-atoms can be thought to be connected by springs with a longitudinal, $L$, and two different transverse spring constants, $T_{1}$ and $T_{2}$, allowing for noncentral forces (Gupta et al., 2001). The spring constants are independent of the types of atoms they connect (homogeneous elasticity). The lattice misfit and the resulting elastic interactions are due to difference in radii of the two types of atoms, $R_{A}>R_{B}$. The Hamiltonian of the model system can be written as a quadratic form of the spin variables $\sigma(\boldsymbol{r})$ and the displacements $v(\boldsymbol{r})$, corresponding to a second-order-expansion treatment of compositional and displacive disorder (Cook and de Fontaine, 1969; Asta and Foiles, 1996). The relaxation time of the lattice distortions is assumed to be much shorter than the diffusion time of the atoms, i.e. the system is always in mechanical equilibrium. Using the Fourier transform of the spin variable, $\tilde{\sigma}(\boldsymbol{k})=\sum_{\boldsymbol{r}} \sigma(\boldsymbol{r}) e^{i \boldsymbol{k} \boldsymbol{r}}$, the Hamiltonian can then be written as (Khachaturyan, 1983),

$$
H=\tilde{H}_{0}+\frac{1}{2 N} \sum_{k \neq 0} B(\boldsymbol{k})|\tilde{\sigma}(\boldsymbol{k})|^{2},
$$

where the sum extends over the first Brillouin zone of the lattice, $\tilde{H}_{0}$ a constant. The "elastic potential" $B(\boldsymbol{k})$ can be calculated for any type of lattice (Cook and de Fontaine, 1969; Gupta et al., 2001). It is important to note that although the interactions are now long-range and anisotropic, they are still describable as pairwise interactions between atoms (Fratzl and Penrose, 1995), i.e.,

$$
H=\tilde{H}_{0}^{\prime}+\frac{1}{2} \sum_{r} \sum_{r^{\prime}}\left(J\left(\boldsymbol{r}-\boldsymbol{r}^{\prime}\right)\right) \sigma(\boldsymbol{r}) \sigma\left(\boldsymbol{r}^{\prime}\right) .
$$

Model parameters like the spring constants can be experimentally obtained (e.g., by neutron scattering) or calculated using more accurate descriptions of the alloy energetics (e.g., the embedded-atom method (Asta and Foiles, 1996)). The effects of an additional external stress can also be modeled within this framework to study directional coarsening and rafting (Section 3.2.2). To observe nontrivial effects, the model has to be modified by including a weak dependence of the spring constants on the atoms connected by the springs (Laberge et al., 1997).

An important parameter defining the elastic properties of the system is the elastic anisotropy $B_{a}$, which is defined as

$$
B_{a}=\frac{c_{11}-c_{12}-2 c_{44}}{2}
$$

In our simulations, we chose a negative elastic anisotropy corresponding to elastically soft $\langle 100\rangle$ directions $(\langle 10\rangle$ direction in the $2 \mathrm{D}$ case). In particular, for the three- 
dimensional model Gupta et al. took the experimentally obtained Born-van Karman parameters of copper to specify the values for the spring constants, $L, T_{1}$, and $T_{2}$. Since introducing a vacancy in a model which allows for lattice distortions is not straightforward, the simulations investigating the influence of elastic interactions have been performed using Kawasaki dynamics (Gupta et al., 2001).

We want to mention an alternative model approach, the so-called Discrete Atom method (Lee, 1998), which assumes central forces, but considers non-homogeneous elasticity. The assumption of harmonic forces can also be suspended (Ikeda and Matsuda, 1993). A comprehensive overview on modeling alloys with misfits including also continuum models can be found in Fratzl et al. (1999).

\subsection{Influence of Elastic Interactions on Phase Separation}

\subsubsection{Varying the Lattice Misfit}

The lattice misfit $\delta$ is defined as $\delta \equiv\left(a_{A}-a_{B}\right) / a_{A}$, where $a_{A}$ and $a_{B}$ are the lattice constants of the pure $A$ and $B$ phases. As a first simulation result has shown that changes in the value of $\delta$, involves also changes of the phase diagram, especially of the critical temperature $T_{c}$. If the elastic interactions, as measured by the size of $\delta$ are increased at fixed value for the parameter $J$ which specify the "chemical" part of the interaction, the effect is to increase the critical temperature for phase separation. This was demonstrated by simulations and the same effect can be seen in the meanfield approximation (Fratzl and Penrose, 1996; Gupta et al., 2001). For order-disorder transitions, on the other hand, the critical temperature is decreased by the elastic interaction, as is the tricritical temperature. This was demonstrated by simulations of Fischer et al. (2000).

Figure 5 summarizes some unpublished results of 3D simulations concerning the influence of different misfits and different concentrations of precipitate atoms on the morphology of the microstructure. For no misfit (Fig. 5, left column) the isolated precipitates are round and irregularly arranged at low concentration and form an interconnected structure at higher concentrations. For $\delta=1 \%$ the precipitates are clearly orientated perpendicular to the elastically soft $\langle 100\rangle$ directions. In the case $c=0.2$ the precipitates have the form of platelets. They are grouped together in domains of platelets with the same orientation. At higher concentration $(c=0.5)$ only a single domain is observed, built up of a series of plates, which have quasi infinite dimensions due to the periodic boundary conditions. For intermediate misfit of $\delta=0.5 \%$ in the microstructure some alignment of the precipitates is combined with more or less irregular features. In agreement with earlier simulations in 2D (Fratzl and Penrose, 1996), the elastic interactions seem more effective for higher concentrations, leading to a more regular microstructure.

To quantify the degree of alignment it is useful to analyze the time-dependent structure function $S(\boldsymbol{k}, t)$ of the precipitate microstructure,

$$
S(\boldsymbol{k}, t)=\frac{1}{N}\left|\sum_{\boldsymbol{r}} \sigma(\boldsymbol{r}, t) e^{i \boldsymbol{k} \boldsymbol{r}}\right|^{2}
$$

i.e., the squared Fourier transform of the atom configuration defined by the spin variable $\sigma(\boldsymbol{r}, t), N$ being the number of lattice sites. Since $S(\boldsymbol{k}, t)$ is measured in a 


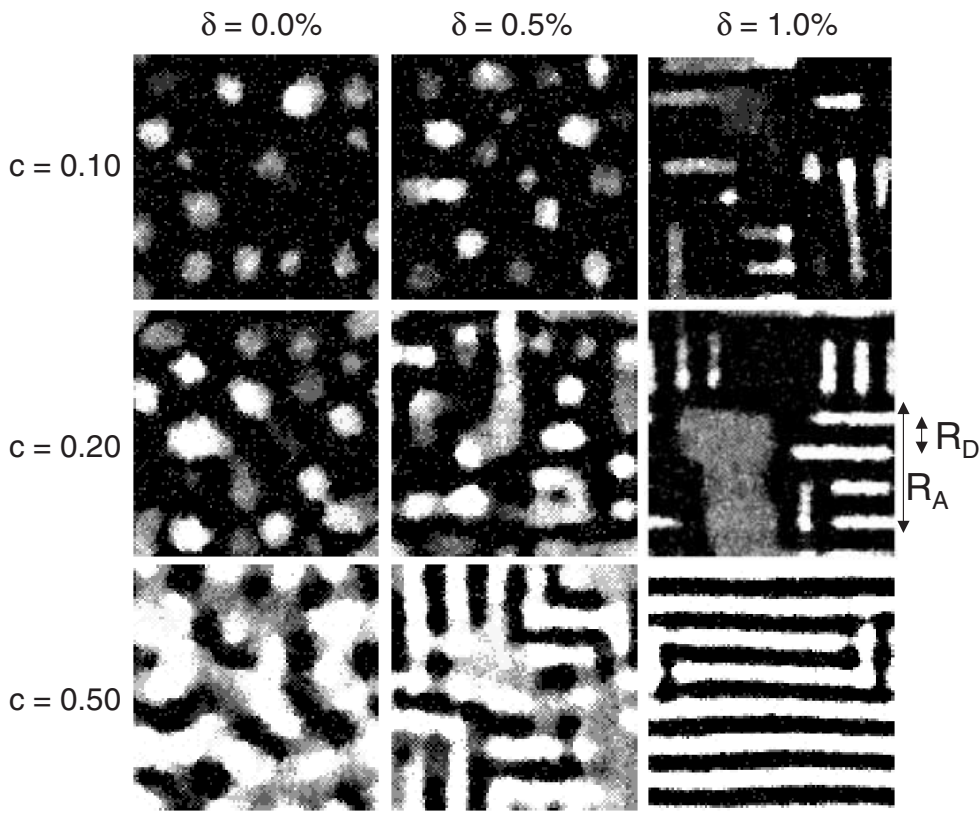

FIGURE 5 Snapshots of the precipitate morphology for three different misfits $\delta$ and three different concentrations of solute atoms $c$ at $t=6000 \mathrm{MCS}$ and $T=5 \mathrm{~J} / \mathrm{k}_{\mathrm{B}}$. The two-dimensional plots were obtained by taking a cross section of a finite thickness equal to 6 lattice constants and averaging along the thickness. Light regions denote regions of a high concentration of solute $A$ atoms. On the right the two different length scales $R_{D}$, the inter-precipitate spacing, and $R_{A}$, the precipitate domain size, are plotted.

single crystal small-angle scattering (SAS) experiment, Fig. 6 corresponds to the scattering pattern obtained with an area detector in a SAS experiment. In the case of no misfit the SAS pattern shows spherical symmetry. For the case $\delta=1 \%$ and $c=0.2$ (middle row on the right in Fig. 5), the alignment of the precipitates is reflected in an anisotropic SAS pattern (Fig. 6), with streaks along the horizontal and vertical directions. Also higher-order maxima can be observed.

To get a quantitative measure of the anisotropy of $S(\boldsymbol{k}, t)$, we compared the fraction of $S(\boldsymbol{k}, t)$ along the axis to the one in off-axis directions, i.e., in 3D we define,

$$
\kappa(t)=\frac{\langle S(\boldsymbol{k}, t)\rangle_{\langle 100\rangle}-\langle S(\boldsymbol{k}, t)\rangle_{\langle 110\rangle \cup\langle 111\rangle}}{\langle S(\boldsymbol{k}, t)\rangle_{\langle 100\rangle \cup\langle 110\rangle \cup\langle 111\rangle}},
$$

where all the points $\boldsymbol{k}$ in the first Brillouin zone are partitioned into groups according to which of the 26 different $\langle 100\rangle,\langle 110\rangle$, and $\langle 111\rangle$-directions they are closest to. $\langle S(\boldsymbol{k}, t)\rangle_{\langle 100\rangle}$ denotes the average value of the structure function in the $\langle 100\rangle$ group. The anisotropy $\kappa(t)$ takes values close to zero in the case of no misfit. In the presence of a misfit, $\kappa(t)$ increases monotonically with time, taking higher values for larger values of the misfit $\delta$ and the concentration $c$.

The structure function can also be used to study the length scales in the system, although the task is not straightforward because the shapes of the precipitates and their alignment and relative positions are not spherically symmetric. The spherically 

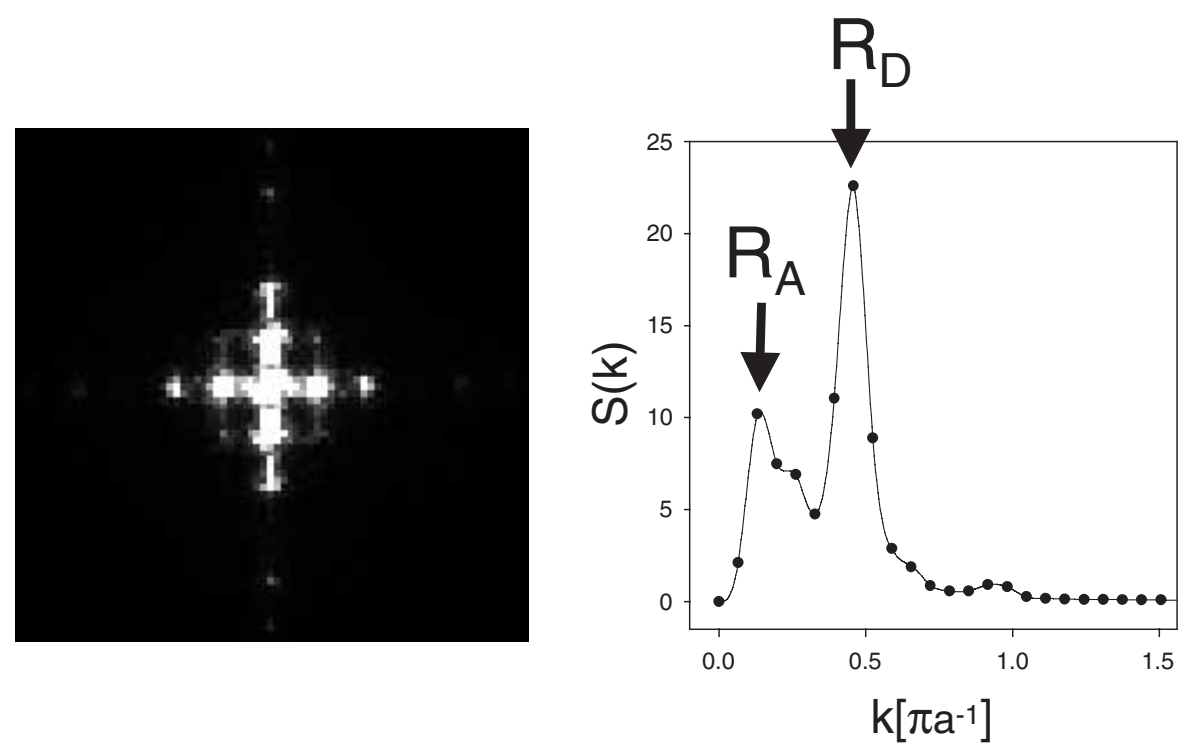

FIGURE $6\langle 100\rangle$ plane of the structure function $S(\boldsymbol{k})$ (left) for misfit $\delta=1 \%$ and concentration $c=0.2$, corresponding to the microstructure shown in Fig. 5. The spherically averaged structure function $S(|\boldsymbol{k}|)$ displays two peaks corresponding to two different length scales in the system; $a$ denotes the cubic lattice constant.

averaged structure function, $S(|\boldsymbol{k}|, t)$ (or one of its higher moments), usually displays a peak, which shifts during coarsening toward smaller values of $|\boldsymbol{k}|$. From the position of the peak, a mean distance $R$ between the precipitates can be derived as, $R(t) \equiv 2 \pi / k_{\max }(t)$. Interestingly, spherically averaging $S(\boldsymbol{k}, t)$ in Fig. 6 results in a function which displays two peaks (Fig. 6, right). Both peaks come from contributions along the $\langle 100\rangle$ directions and one peak is not simply a higher-order peak of the other. There is a peak at $k=2 \pi / R_{D}$, where $R_{D}$ is the distance between the precipitates, but there is a second peak at a position $k=2 \pi / R_{A}$; the value of $R_{A}$ is about the same as the size of the domains of parallel precipitates that are visible in Fig. 5. Having defined these two length scales, the question follows how they evolve in time. Again, $R_{D}(t)$ and $R_{A}(t)$ are plotted on the natural timescale, $t^{1 / 3}$ (Fig. 7). For zero misfit the data for the inter-precipitate spacing $R_{D}(t)$ can be well fitted by a straight line. However, with increasing misfit, the kinetics slows down, with the curve bending the earlier the larger the misfit. For $\delta>1$ coarsening comes virtually to rest. The upper part of Fig. 7 shows how the precipitate domains grow in time. Since their size is close to the length of the whole system, finite size effects will have a considerable influence on the result. From making just a qualitative statement, we observe in our simulations for large lattice misfit a coarsening process on two length scales.

As a last result, we want to report about the influence of a lattice misfit on the precipitate size distribution. Looking at the scaled precipitate size distribution, i.e. the sizes of the precipitates were normalized by the mean precipitate size, we observed a broadening of the distribution for $\delta>0.5$ (Weinkamer et al., 2001). While for no misfit the microstructure consisted of isolated precipitates at a concentration of solute atoms $c=0.2$, a high misfit led to a percolated network-like precipitate structure. Our interpretation is that the nonspherical shape of the precipitates, their 


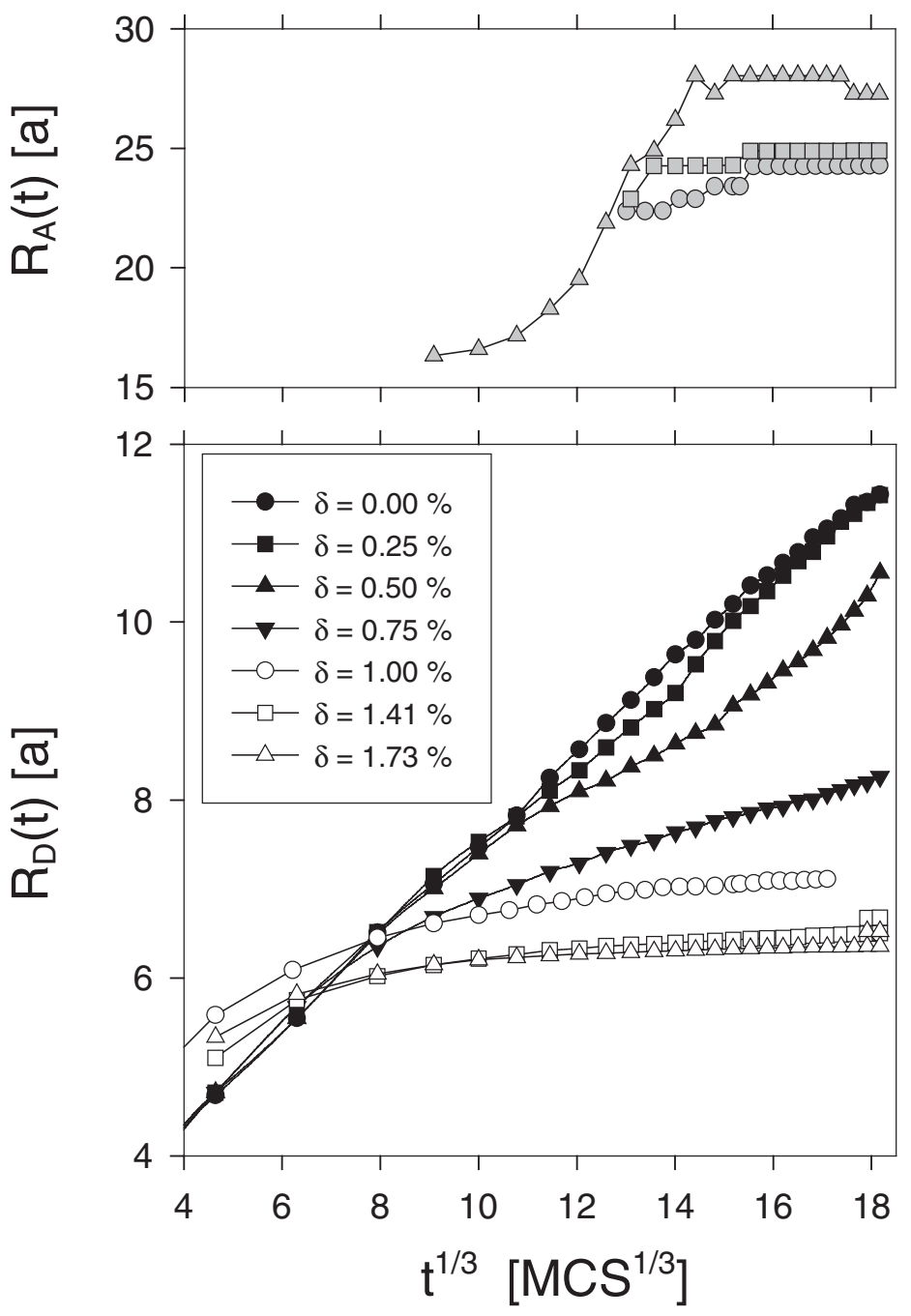

FIGURE 7 Time evolution of the inter-precipitate spacing $R_{D}$ on a $t^{1 / 3}$ scale for concentration $c=0.2$, in units of the cubic lattice constant $a$. For large misfits $\delta \geq 1 \%, R_{A}$ could be defined corresponding to the domain size of precipitates with the same orientation (plot above with gray symbols).

orientation perpendicular to the $\langle 100\rangle$ directions, and their regular arrangement, results in a higher probability of a coalescence between precipitates.

\subsubsection{Applying an Additional External Load-Precipitate Rafting}

From real alloys, it is known that the interference of an externally applied load with an internal stress state due to a lattice misfit between the phases causes new effects during phase separation. Most strikingly the microstructure "follows" the orientation impressed by a uniaxial load. Early simulations in two dimensions demonstrated that the emerging precipitate morphology exhibited a preferred orientation. Wavy stripes developed and aligned parallel to the axis of the uniaxial tensile load (Laberge et al., 


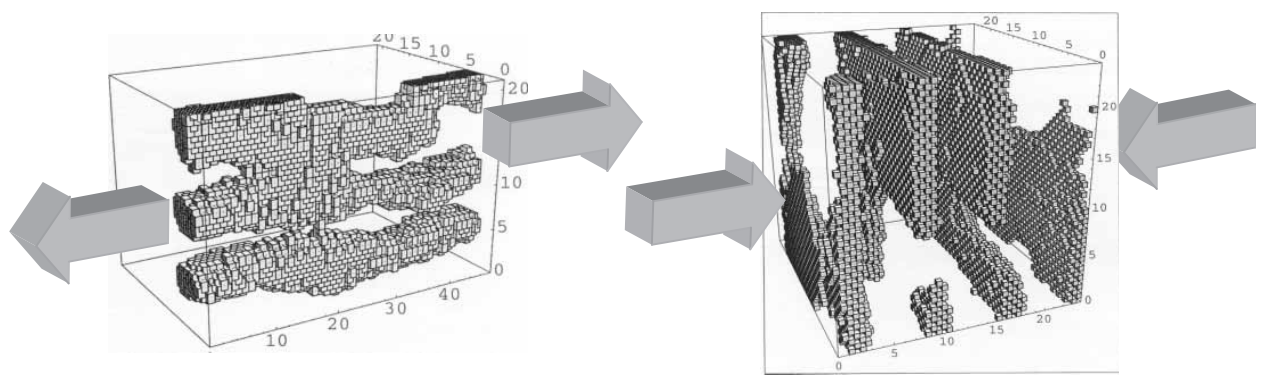

FIGURE 8 Snapshots of the precipitate morphology for an alloy evolved from a random configuration at $t=4000 \mathrm{MCS}$ for external tensile stress and compressive stress as indicated by the arrows $(c=0.2$ and $T=5 \mathrm{~J} / \mathrm{k}_{\mathrm{B}}$ ). Lattice sites occupied by solute $A$ atoms are represented by cubes. $B$ atoms and $A$ atoms dissolved in the matrix are not drawn. In each case only a small part of the whole system is shown.

1995, 1997). The drawback of these simulations is that there is no direct conclusion from a stripe-like pattern in two dimensions to the microstructure in $3 \mathrm{D}$.

Predicting the rafting morphology in an alloy with negative anisotropy needs the consideration of (i) the sign of the misfit, i.e., is the lattice spacing of the precipitate phase larger or smaller, (ii) the sign of the elastic inhomogeneity, i.e., is the precipitate phase harder or softer, and (iii) the sign of the applied load, i.e., tensile or compressive. In our $3 \mathrm{D}$ simulations, we investigated the case of a larger lattice misfit $(\delta=1 \%)$ and larger elastic constants in the precipitates. In agreement with theoretical considerations (Socrate and Parks, 1993) cylindrical precipitates along the loading direction are formed when applying a tensile load and plate-like precipitates perpendicular to the loading direction in the case of a compressive load (Gupta et al., 2001) (Fig. 8). In the framework of our model, the change of one of the three signs mentioned above, e.g., changing the sign of the lattice misfit, would result in a switch from the cylindrical to the plate-like morphology or vice versa. It is notable that the precipitates are far from being perfect plates or cylinders, but they are partly interconnected, showing holes and other irregularities (Fig. 8).

In analogy with the introduction of the anisotropy parameter $\kappa(t)$ (Eq. (19)), we used again the structure function $S(\boldsymbol{k}, t)$ as defined in Eq. (18) to quantify the asymmetry of the microstructure with respect to the loading direction and directions perpendicular to it. The definition of the asymmetry parameter $\eta_{[100]}(t)$ reads as,

$$
\eta_{[100]}(t)=\frac{\langle S(\boldsymbol{k}, t)\rangle_{[100]}}{\langle S(\boldsymbol{k}, t)\rangle_{[100]}+\langle S(\boldsymbol{k}, t)\rangle_{[010]}+\langle S(\boldsymbol{k}, t)\rangle_{[001]}}
$$

with the same notation as in Eq. (18), but with a separate evaluation of the three $\langle 100\rangle$ directions. Analogous parameters are defined for the [010] and the [001] directions. For a compressive load along the [100] direction, $\eta_{[100]}(t)$ approached 1 , while both other components took values close to zero at later times. As expected from the cylindrical form of the precipitates in the case of tensile stress, the two components normal to the load axis increased and the parallel component decreased. With the asymmetry parameters, we could demonstrate that the application of an external load leads from the very beginning of phase separation to a distinction of the loading direction. 
Reversing the external stress from tensile to compressive or vice versa, caused only very slow changes in the asymmetry parameters. The parallel-cylinder morphology remained fairly stable when a compressive load was applied (Gupta et al., 2001).

To estimate the size of the precipitates, the total interface present in the system was used. With the definition

$$
R_{S}=\frac{12 N c}{N_{A B}} a
$$

with $a$ the lattice parameter, $c$ the concentration of solute atoms, $N$ the number of lattice sites, and $N_{A B}$ the total number of $A-B$ bonds, $R_{S}$ equals the edge length in the case of cubic precipitates. The time evolution of $R_{S}$ was not only very similar regardless of the sign of the applied load, but also of whether an additional load was applied at all. This independence of $R_{S}(t)$ on the external stress agreed with results of simulations in $2 \mathrm{D}$. In contrast to earlier $2 \mathrm{D}$ results, however, $R_{S}(t)$ could not be fitted by a law of the form given by Eq. (13). As already shown for the length scale defined via the spherically averaged structure function $R_{D}(t)$ (Fig. 7), $R_{S}(t)$ also slows down significantly (Gupta et al., 2001).

Our explanation of this reduced growth rate is based on the regularity of the emerging microstructure. In the case of a tensile load, the cylindrical precipitates are arranged in a regular two-dimensional array, forming a quadratic mesoscopic precipitate lattice (Fig. 9). For a compressive load the plate-like precipitates form a onedimensional array. Once such a regular arrangement of precipitates was formed it is hard to imagine how coarsening should proceed at all. For example, how is in the tensile case the number of precipitates reduced, say from 49 cylindrical precipitates arranged in a $7 \times 7$ array to 36 forming a $6 \times 6$ array. Our simulations showed that the mesoscopic precipitate lattices are not perfect, but contain mesoscopic lattice defects (Weinkamer et al., 2000). In analogy to defects in ordinary crystals, the defects can be classified as "meso-vacancies", "meso-interstitials", and "meso-dislocations". These defects of the precipitate lattice are involved in complex dynamical processes, which decrease the number of precipitates and consequently increase the lattice constant of the mesoscopic lattice: meso-dislocations can move against each other and annihilate (Fig. 9), meso-interstitials can merge with a precipitate occupying a regular site of the meso-lattice. The series of plate-like precipitates in the compressive case exhibited a mesoscopic analog to a stacking fault in crystal lattices (Weinkamer et al., 2000).

\subsubsection{Ordered Precipitates}

Choosing antiferromagnetic $\mathrm{NN}$ interactions, $J^{(1)}>0$, and ferromagnetic next $\mathrm{NN}$ neighbour interactions, $J^{(2)}<0$, results in an ordering tendency. The joint ordering and decomposition were first investigated using a conventional Ising model with a rigid lattice (Gorentsveig et al., 1997). The inclusion of elastic interactions into a two-dimensional model (Nielaba et al., 1999) leads to the development of a stripelike morphology for the segregated ordered and disordered regions. A new morphological feature is the presence of narrow channels subdividing the ordered plates. These channels can be explained by the possibility of anti-phase boundaries (APBs) separating the two variants of the ordered phase. Since it is commonly found that neighbouring 


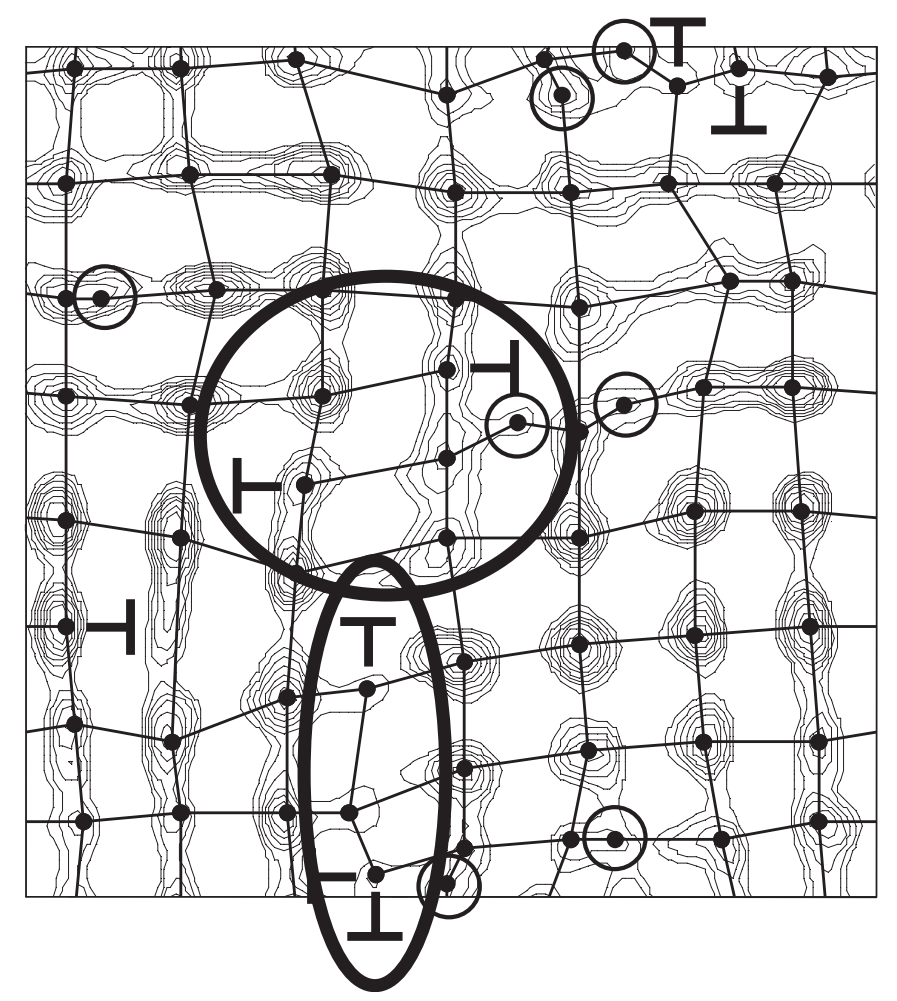

FIGURE 9 Contour plot of the concentration of solute $A$ atoms averaged along the direction of tensile stress, i.e., normal to the plotting plane at $t=1200 \mathrm{MCS}$. Peak values of the concentration, marked by a full circle, are connected to emphasize the quadratic "meso-lattice" of the precipitates. Defects like meso-dislocations $(\perp)$ and meso-interstitials $(\bigcirc)$ are labeled. The two regions highlighted by the black ellipses show proximate meso-dislocations which move toward each other and annihilate eventually.

precipitates are ordered on different sublattices, the narrow channels correspond to wetted APBs. The great difference in the morphology with $30 \%$ ordered phase in the disordered matrix compared to the "reverse case" with $30 \%$ disordered phase is as well attributed to the presence of APBs.

The effect of an additional external stress is to cause rafting of the ordered precipitates. Because of the APBs the stripes are then segmented. The simulation results are best compared to the technologically important class of nickel-base superalloys where the precipitates are also ordered. Figure 10 shows transmission electron micrographs of Ni-Al-Mo alloys annealed with and without external stress and simulation results. Despite the great difference in the length scale, the great similarity between the real and the computed morphologies is striking.

\section{CONCLUSIONS}

The kinetic Ising model and its extensions to the ABV model or to include an elastically deformable lattice is able to predict a number of important details of phase separation which depend on processes on the atomic level. While these microscopic models are probably too detailed to perform large-scale simulations of real alloys, they provide 


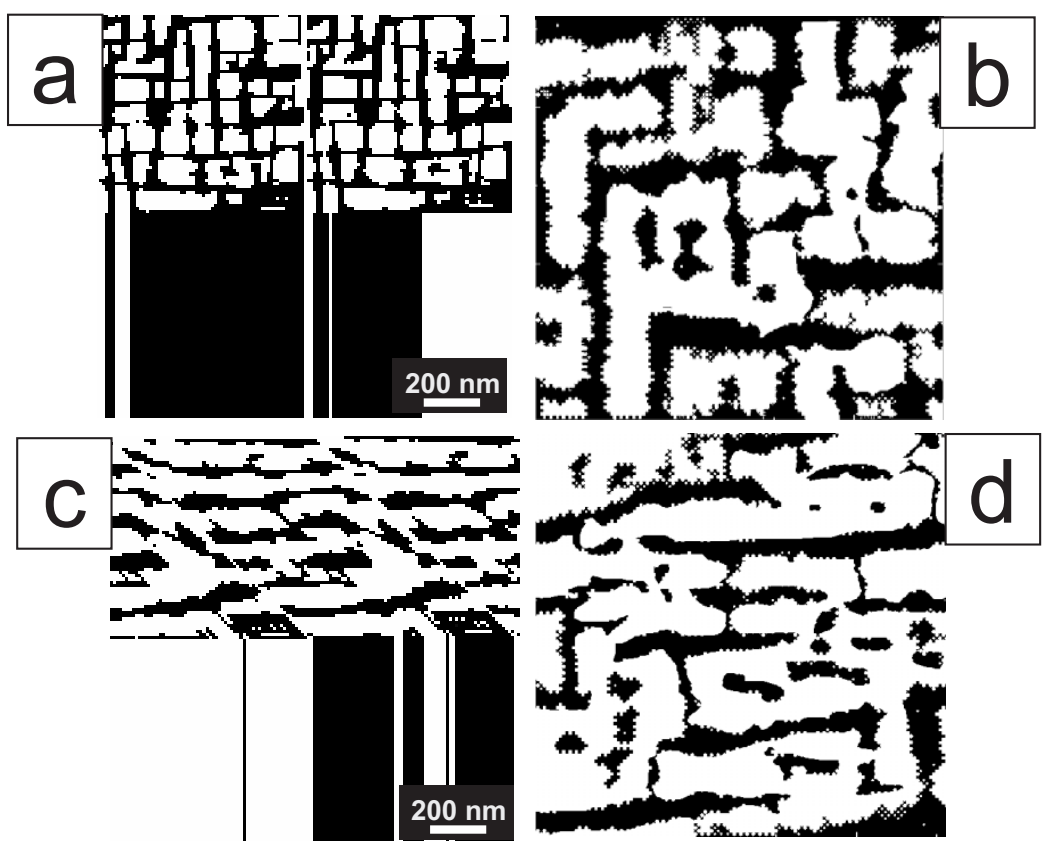

FIGURE 10 Comparison between transmission electron micrographs and simulation snapshots. The disordered phase is shown black, while all ordered variants are shown in white. Plots (a) and (c) on the left are TEM images of $\mathrm{Ni}-\mathrm{Al}-\mathrm{Mo}$ alloy (from Paris et al., 1997) without external load (a) and with an external load along the vertical [010] direction (c). The simulations were performed at a concentration $c=0.35$ and temperature $T=0.567 \mathrm{~J} / \mathrm{k}$. Snapshots are taken after $10^{6} \mathrm{MCS}$, without (b) and with (d) including an external stress.

basic knowledge complementary to the results which may be obtained by mesoscopic or macroscopic models of phase separation.

\section{References}

Abinandanan, T.A., Haider, F. and Martin, G. (1998). Computer simulations of diffusional phase transformations: Monte Carlo algorithm and application to precipitation of ordered phases. Acta Materialia, 46, 4243.

Aspelmeier, T., Schmittmann, B. and Zia, R.K.P. (2001). Microscopic kinetics and time-dependent structure factors. Physical Review Letters, 8706, art. no.

Asta, M. and Foiles, S.M. (1996). Embedded-atom-method effective-pair-interaction study of the structural and thermodynamic properties of $\mathrm{Cu}-\mathrm{Ni}, \mathrm{Cu}-\mathrm{Ag}$, and $\mathrm{Au}-\mathrm{Ni}$ solid solutions. Physical Review B, 53, 2389.

Athenes, M., Bellon, P. and Martin, G. (1997). Identification of novel diffusion cycles in B2 ordered phases by Monte Carlo simulation. Philosophical Magazine a-Physics of Condensed Matter Structure Defects and Mechanical Properties, 76, 565.

Athenes, M., Bellon, P. and Martin, G. (2000). Effects of atomic mobilities on phase separation kinetics: A Monte-Carlo study. Acta Materialia, 48, 2675.

Athenes, M., Bellon, P., Martin, G. and Haider, F. (1996). A Monte-Carlo study of B2 ordering and precipitation via vacancy mechanism in BCC lattices. Acta Materialia, 44, 4739.

Bellon, P. (2003). Kinetic Monte Carlo simulations in crystalline alloys: principles and selected applications. NATO Sci. Ser. II Math., Thermodynamics, Microstructures and Plasticity. A. e. a. Finel, Kluwer Academic: 395.

Binder, K. (1997). Applications of Monte Carlo methods to statistical physics. Reports on Progress in Physics, 60, 487. 
Binder, K. and Fratzl, P. (2001). Phase Transformations in Materials. G. Kostorz, Wiley-VCH: Chapter 6.

Binder, K. and Heermann, D.W. (2002). Monte Carlo Simulation in Statistical Physics: An Introduction. Springer, Berlin, New York.

Binder, K. and Stauffer, D. (1974). Theory for slowing down of relaxation and spinodal decomposition of binary-mixtures. Physical Review Letters, 33, 1006.

Bortz, A.B., Kalos, M.H. and Lebowitz, J.L. (1975). New algorithm for Monte-Carlo simulation of Ising spin systems. Journal of Computational Physics, 17, 10.

Bortz, A.B., Kalos, M.H., Lebowitz, J.L. and Zendejas, M.A. (1974). Time evolution of a quenched binary alloy - computer-simulation of a 2-dimensional model system. Physical Review B, 10, 535.

Chen, L.Q. (2002). Phase-field models for microstructure evolution. Annual Review of Materials Research, 32, 113.

Cook, H.E. and de Fontaine, D. (1969). On elastic free energy of solid solutions. I. Microscopic Theory. Acta Metallurgica, 17, 915.

Fischer, D. and Nielaba, P. (2000). Phase diagram of a model alloy with lattice misfit. Physica A, $279,287$.

Fratzl, P. and Penrose, O. (1994). Kinetics of spinodal decomposition in the Ising-model with vacancy diffusion. Physical Review B, 50, 3477.

Fratzl, P. and Penrose, O. (1995). Ising-model for phase-separation in alloys with anisotropic elastic interaction. 1. Theory. Acta Metallurgica Et Materialia, 43, 2921.

Fratzl, P. and Penrose, O. (1996). Ising model for phase separation in alloys with anisotropic elastic interaction. 2. A computer experiment. Acta Materialia, 44, 3227.

Fratzl, P. and Penrose, O. (1997). Competing mechanisms for precipitate coarsening in phase separation with vacancy dynamics. Physical Review B, 55, R6101.

Fratzl, P., Lebowitz, J.L., Penrose, O. and Amar, J. (1991). Scaling functions, self-similarity, and the morphology of phase-separating systems. Physical Review B, 44, 4794.

Fratzl, P., Penrose, O. and Lebowitz, J.L. (1999). Modeling of phase separation in alloys with coherent elastic misfit. Journal of Statistical Physics, 95, 1429.

Fratzl, P., Penrose, O., Weinkamer, R. and Zizak, I. (2000). Coarsening in the Ising model with vacancy dynamics. Physica A, 279, 100.

Frontera, C., Vives, E., Castan, T. and Planes, A. (1997). Monte Carlo study of the growth of L1(2)-ordered domains in FCC A(3)B binary alloys. Physical Review B, 55, 212.

Gerold, V. and Kern, J. (1987). The determination of atomic interaction energies in solid-solutions from short-range order coefficients - an inverse Monte-Carlo method. Acta Metallurgica, 35, 393.

Gorentsveig, V.I., Fratzl, P. and Lebowitz, J.L. (1997). Kinetics of joint ordering and decomposition in binary alloys. Physical Review B, 55, 2912.

Gupta, H., Weinkamer, R., Fratzl, P. and Lebowitz, J.L. (2001). Microscopic computer simulations of directional coarsening in face-centered cubic alloys. Acta Materialia, 49, 53.

Heermann, D.W., Li, Y.X. and Binder, K. (1996). Scaling solutions and finite-size effects in the LifshitzSlyozov theory. Physica A, 230, 132.

Huse, D.A. (1986). Corrections to late-stage behaviour in spinodal decomposition - Lifshitz-Slyozov scaling and Monte-Carlo simulations. Physical Review B, 34, 7845.

Ikeda, H. and Matsuda, H. (1993). Effects of difference in Elastic-Moduli between constituents on spinodal decomposition processes. Materials Transactions Jim, 34, 651.

Iwai, T. (1999). Vacancy-mediated phase separation of binary alloy. Journal of the Physical Society of Japan, 68, 3717.

Kantelhardt, J.W., Koscielny-Bunde, E., Rego, H.H.A., Havlin, S. and Bunde, A. (2001). Detecting long-range correlations with detrended fluctuation analysis. Physica A, 295, 441.

Khachaturyan, A.G. (1983). Theory of Structural Transformations in Solids. Wiley, New York.

Kikuchi, R. and Sato, H. (1972). Diffusion-coefficient in an ordered binary alloy. Journal of Chemical Physics, 57, 4962.

Laberge, C.A., Fratzl, P. and Lebowitz, J.L. (1995). Elastic effects on phase segregation in alloys with external stresses. Physical Review Letters, 75, 4448.

Laberge, C.A., Fratzl, P. and Lebowitz, J.L. (1997). Microscopic model for directional coarsening of precipitates in alloys under external load. Acta Materialia, 45, 3949.

Le Bouar, Y. and Soisson, F. (2002). Kinetic pathways from embedded-atom-method potentials: Influence of the activation barriers. Physical Review B, 65, 094103.

Lebowitz, J.L., Marro, J. and Kalos, M.H. (1982). Dynamical scaling of structure-function in quenched binary-alloys. Acta Metallurgica, 30, 297.

Lee, J.K. (1998). Elastic stress and microstructural evolution. Materials Transactions Jim, 39, 114.

Lengeler, B. (2001). Coherence in X-ray physics. Naturwissenschaften, 88, 249.

Marro, J., Bortz, A.B., Kalos, M.H. and Lebowitz, J.L. (1975). Time evolution of a quenched binary alloy 2. Computer-simulation of a 3-dimensional model system. Physical Review B, 12, 2000.

Marro, J., Lebowitz, J.L. and Kalos, M.H. (1979). Computer-simulation of the time evolution of a quenched model alloy in the nucleation region. Physical Review Letters, 43, 282. 
Mukherjee, S. and Cooper, B.R. (1998). Coarsening in the presence of vacancies. Materials Science and Engineering a-Structural Materials Properties Microstructure and Processing, 248, 101.

Nielaba, P., Fratzl, P. and Lebowitz, J.L. (1999). Growth of ordered domains in a computer model alloy with lattice misfit. Journal of Statistical Physics, 95, 23.

Novotny, M.A. (1995). A new approach to an old algorithm for the simulation of Ising-like systems. Computers in Physics, 9, 46.

Pareige, C., Soisson, F., Martin, G. and Blavette, D. (1999). Ordering and phase separation in Ni-Cr-Al: Monte Carlo simulations vs three-dimensional atom probe. Acta Materialia, 47, 1889.

Paris, O., Fahrmann, M., Fahrmann, E., Pollock, T.M. and Fratzl, P. (1997). Early stages of precipitate rafting in a single crystal Ni-Al-Mo model alloy investigated by small-angle X-ray scattering and TEM. Acta Materialia, 45, 1085.

Porta, M. and Castan, T. (1996). Monte Carlo study of the domain growth in nonstoichiometric twodimensional binary alloys. Physical Review B, 54, 166.

Porta, M., Frontera, C., Vives, E. and Castan, T. (1997). Effect of the vacancy interaction on antiphase domain growth in a two-dimensional binary alloy. Physical Review B, 56, 5261.

Porta, M., Vives, E. and Castan, T. (1999). Vacancy-assisted domain growth in asymmetric binary alloys: A Monte Carlo study. Physical Review B, 60, 3920.

Puri, S. (1997). Dynamics of vacancy-mediated phase separation. Physical Review E, 55, 1752.

Puri, S. and Sharma, R. (1998). Phase ordering dynamics in binary mixtures with annealed vacancies. Physical Review E, 57, 1873.

Rao, M., Kalos, M.H., Lebowitz, J.L. and Marro, J. (1976). Time evolution of a quenched binary alloy 3. Computer-simulation of a 2-dimensional model system. Physical Review B, 13, 4328.

Rautiainen, T.T. and Sutton, A.P. (1999). Influence of the atomic diffusion mechanism on morphologies, kinetics, and the mechanisms of coarsening during phase separation. Physical Review B, 59, 13681.

Roussel, J.M. and Bellon, P. (2001). Vacancy-assisted phase separation with asymmetric atomic mobility: Coarsening rates, precipitate composition, and morphology. Physical Review B, 6318, art. no.

Schmittmann, B. and Thies, M. (2002). Vacancy-mediated domain growth in a driven lattice gas. Europhysics Letters, 57, 178.

Schweika, W. (1998). Disordered Alloys: Diffuse Scattering and Monte Carlo Simulations. Springer, Berlin, New York.

Socrate, S. and Parks, D.M. (1993). Numerical determination of the elastic driving force for directional coarsening in Ni-superalloys. Acta Metallurgica Et Materialia, 41, 2185.

Soffa, W.A., Puschl, W. and Pfeiler, W. (2003). Homo or hetero? How vacancy motion brings discreteness into phase transformations. Intermetallics, 11, 161.

Soisson, F. and Martin, G. (2000). Monte Carlo simulations of the decomposition of metastable solid solutions: Transient and steady-state nucleation kinetics. Physical Review B, 62, 203.

Stadler, L.-M., Sepiol, B., Weinkamer, R., Hartmann, M., Fratzl, P., Kantelhardt, J.W., Zotone, F., Grübel, G. and Vogl, G. (2003). Long-term correlations distinguish coarsening mechanisms in alloys. Physical Review B, 68, 180101R.

Sur, A., Lebowitz, J.L., Marro, J. and Kalos, M.H. (1977). Time evolution of a quenched binary alloy 4. Computer-simulation of a 3-dimensional model system. Physical Review B, 15, 3014.

Thornton, K., Agren, J. and Voorhees, P.W. (2003). Modelling the evolution of phase boundaries in solids at the meso- and nanoscales. Acta Materialia, 51, 5675.

Vives, E. and Planes, A. (1992). Kinetics of a vacancy-driven order-disorder transition in a 2-dimensional binary alloy. Physical Review Letters, 68, 812.

Vives, E. and Planes, A. (1993). Ordering kinetics by vacancies. International Journal of Modern Physics C-Physics and Computers, 4, 701.

Wagner, R., Kampmann, R. and Voorhees, P.W. (2001). Phase Transformations in Materials. G. Kostorz, Wiley-VCH: Chapter 5.

Weinkamer, R. and Fratzl, P. (2003). By which mechanism does coarsening in phase-separating alloys proceed? Europhysics Letters, 61, 261.

Weinkamer, R., Fratzl, P., Sepiol, B. and Vogl, G. (1998). Monte Carlo simulation of diffusion in a B2-ordered model alloy. Physical Review B, 58, 3082.

Weinkamer, R., Fratzl, P., Sepiol, B. and Vogl, G. (1999). Monte Carlo simulations of Mossbauer spectra in diffusion investigations. Physical Review B, 59, 8622.

Weinkamer, R., Gupta, H., Fratzl, P. and Lebowitz, J.L. (2000). Dynamics of mesoscopic precipitate lattices in phase-separating alloys under external load. Europhysics Letters, 52, 224.

Weinkamer, R., Gupta, H., Lebowitz, J.L. and Fratzl, P. (2001). Precipitate size distribution in alloys with and without lattice misfit. Zeitschrift Fur Metallkunde, 92, 9.

Wolverton, C., Zunger, A. and Schonfeld, B. (1997). Invertible and non-invertible alloy Ising problems. Solid State Communications, 101, 519.

Yaldram, K. and Binder, K. (1991a). Monte-Carlo simulation of phase-separation and clustering in the ABV model. Journal of Statistical Physics, 62, 161. 
Yaldram, K. and Binder, K. (1991b). Spinodal decomposition of a 2-dimensional model alloy with mobile vacancies. Acta Metallurgica Et Materialia, 39, 707.

Yaldram, K. and Binder, K. (1991c). Unmixing of binary-alloys by a vacancy mechanism of diffusion a computer-simulation. Zeitschrift Fur Physik B-Condensed Matter, 82, 405.

Zhao, L., Najafabadi, R. and Srolovitz, D.J. (1996). Determination of vacancy and atomic diffusivities in solid solution alloys. Acta Materialia, 44, 2737.

Zizak, I., Fratzl, P. and Penrose, O. (1997). Dissolution of precipitates heated above the solubility line: a Monte Carlo simulation. Physical Review B, 55, 12121. 